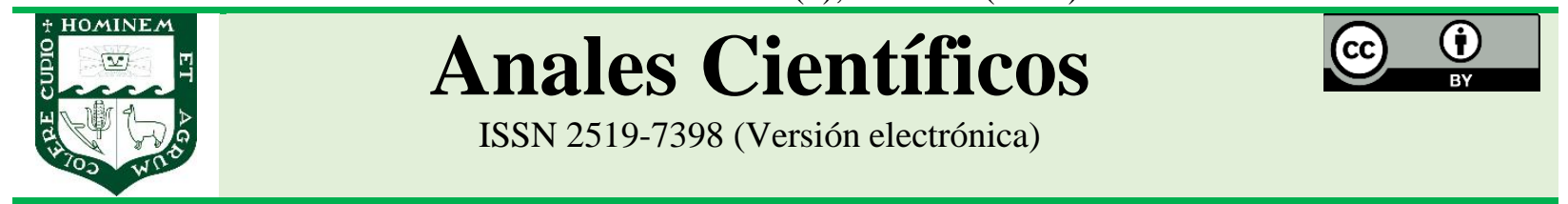

ARTÍCULO ORIGINAL - RESEARCH ARTICLE

http://dx.doi.org/10.21704/ac.v81i2.1667

\title{
EFECTO DEL ABONO ORGÁNICO ACELERADO EN PLANTONES DE CAFÉ (Coffea arabica $\mathbf{L}$.)
}

\section{Effect of accelerated organic fertilizer on coffee seedlings (Coffea arabica L.)}

\author{
Roberto Cosme De La Cruz ${ }^{*}$ (D); Auristela Reynoso Zárate ${ }^{1}$; Enrique Raúl Adama Rojas²; Vicente \\ Pocomucha Poma ${ }^{3}$
}

\footnotetext{
${ }^{1}$ Instituto Nacional de Innovación Agraria, Lima, Perú. ${ }^{2}$ Universidad Nacional Agraria La Molina, 15024, Lima, Perú.

${ }^{2}$ Universidad Nacional Agraria de la Selva, Perú.

* E-mail: rcosme@inia.gob.pe
}

Recibido: 25/05/2020; Aceptado: 15/12/2020; Publicado: 30/12/2020

\begin{abstract}
The objective of the research was to evaluate the effect of accelerated organic fertilizer on the growth and development of coffee plants (Coffea arabica L.), Catimor variety, in the Greenhouse of the Experimental Center and Multipurpose Laboratory of the Instituto Nacional de Innovación Agraria (INIA), district La Molina, department of Lima, Peru. The completely random design was used, with two treatments: treatment 1 (without accelerated organic fertilizer) and treatment 2 (with accelerated organic fertilizer). The following were evaluated: plant height, number of branches, stem diameter, leaf width and length, percentage of dry matter, nitrogen concentration, phosphorus, potassium and calcium in the leaves. The results showed that the use of accelerated organic fertilizer in coffee plants stimulates the variables plant height $(24,76 \mathrm{~cm})$, stem diameter $(5,74 \mathrm{~mm})$, nitrogen concentration $(2,33 \%)$, phosphorus concentration $(0,17 \%)$, calcium concentration $(1,61 \%)$, but without statistical difference between treatments. In the number of branches per plant, the treatment with accelerated organic fertilizer had the highest number of branches with 3,25 branches per plant, compared to the treatment without accelerated organic fertilizer with 1,5 branches per plant.
\end{abstract}

Keywords: Coffe; fertilizer; agriculture; sustainable performance; organic

RESUMEN

El objetivo de la investigación fue evaluar el efecto del abono orgánico acelerado en el crecimiento y desarrollo de plantas de café (Coffea arabica L.), variedad Catimor, en el Invernadero del Centro Experimental La Molina y Laboratorio Multipropósito del Instituto Nacional de Innovación Agraria (INIA), distrito La Molina, departamento de Lima, Perú. Se utilizó el diseño completamente al Azar, con dos tratamientos: tratamiento 1 (sin abono orgánico acelerado) y tratamiento 2 (con abono orgánico acelerado). Se evaluaron: altura de la planta, número ramas, diámetro del tallo, ancho y longitud de hoja, porcentaje de materia seca, concentración de nitrógeno, fósforo, potasio y calcio en las hojas. Los resultados mostraron que el uso de abono orgánico acelerado en plantas de café estimula las variables altura de planta $(24,76 \mathrm{~cm})$, diámetro de tallo $(5,74 \mathrm{~mm})$, concentración de nitrógeno $(2,33 \%)$, 
concentración de fósforo $(0,17 \%)$, concentración de calcio $(1,61 \%)$, pero sin diferencia estadística entre tratamientos. En el número de ramas por planta, el tratamiento con abono orgánico acelerado presentó el mayor número de ramas con 3,25 ramas por planta, en comparación al tratamiento sin abono orgánico acelerado con 1,5 ramas por planta.

Palabras clave: café; fertilizante; agricultura, sostenible; orgánico

Forma de citar el artículo (Formato APA):

Cosme, R., Reynoso, A., Adama, R., \& Pocomucha, V. (2020). Efecto del abono orgánico acelerado en plantones de café (Coffea arabica L.). Anales Científicos. 81(2), 376-384. http://dx.doi.org/10.21704/ac.v81i2.1667

Autor de correspondencia (*): Roberto Cosme De La Cruz. Email: rcosme@inia.gob.pe

(C) Los autores. Publicado por la Universidad Nacional Agraria La Molina.

This is an open access article under the CC BY

\section{INTRODUCCIÓN}

El café, es un producto de origen agrícola de gran importancia internacional. Existen entre unas 100 especies del género Coffea (Davies et al., 2006), solo $C$. arabica L. (café arábico) y C. canephora Pierre ex A. Froehner (café robusto) son económicamente importante a nivel mundial. Los principales países productores del grano tipo arábica y robusta son Brasil (Lammel et al., 2015), Vietnam, Indonesia, Colombia y Etiopia. Es el segundo producto de importancia internacional después del café (DaMatta et al., 2007), el $50 \%$ de la producción del mundo es destinado a la fabricación de café soluble (Ballesteros et al., 2014). Los países productores de café muestran cada vez más interés en comprender los factores que influyen calidad de bebidas (Avelino et al., 2005). En el Perú, el café es un cultivo de gran importancia económica y social; es el principal producto de la agro exportación, el $95 \%$ de la producción nacional se exporta como materia prima y el otro 5\% es para consumo interno de los peruanos (Choquehuanca, 2018).

Las variables que influyen en la producción de café es el genotipo, su adaptación al ambiente depende la cantidad y calidad del fruto a cosechar (Kathurima et al., 2009). Otra variable de gran importancia es el manejo agronómico, dentro de esta última se encuentra la fertilización. De acuerdo con Sadeghian y González (2012) la fertilización busca mantener e incrementar la materia orgánica, nutrientes en el suelo, resistencia de las plantas a condiciones de estrés como la incidencia de plagas, enfermedades y sequías.

Existen diversos estudios relacionados con el efecto de los nutrientes en el cultivo del café, algunos autores mencionan el momento oportuno de realizar la fertilización. Castro-Tanzi (2017) indica que la producción intensiva de café (Coffea arabica) demanda cantidades considerables de potasio $(\mathrm{K})$ y nitrógeno (N). No obstante, los nutrientes son fundamentales para el desarrollo adecuado del cafeto y el desempeño esperado del cultivo (Wang et al., 2015; Kadri et al., 2016). Según Salazar y Sadeghian (2016) la demanda de nutrientes varía con la etapa de desarrollo del cultivo. El desarrollo vegetativo y el fruto requieren considerables cantidades de Nitrógeno $(\mathrm{N})$ y Potasio (K) (Ramírez et al., 2002). López et al. (2016) manifiesta que el $90 \%$ de los productores en México no tienen la cultura de aplicar nutrientes al suelo y los fertilizantes que utilizan carecen de sustento técnico para determinar los nutrientes, cantidades, épocas y las fuentes que deben utilizar para su aplicación. Adicional a ello, los daños ocasionados por la roya en los últimos años, han ocasionado que los productores mejoren sus prácticas de manejo, especialmente la fertilización. Fertilizar incrementa la producción en comparación con el tratamiento sin fertilización. Albaugh et al. (2015) concluyen que el uso de fertilizantes mejora el crecimiento de plantas. Por el incremento de la actividad microbiana mientras los bioestimulantes influyen en el rendimiento y tamaño de fruto (Corral et al., 2016). Siendo común observar agroecosistemas de café donde se aplican cantidades considerables de fertilizantes compuestos (Castro-Tanzi et al., 2012).

En términos prácticos, el manejo de la nutrición en la etapa de crecimiento vegetativo de las plantas de café, inicia desde el transplante del almacigo en el campo y finaliza al inicio de la cosecha (Sadeghian y González, 2012). Para garantizar una producción sostenible se debe dar también prioridad al manejo dentro del vivero; por ello, la necesidad de estudiar la producción adecuada de plantas de café en almacigo, mediante 
pruebas de fertilización con abonos orgánicos acelerados. El objetivo de este estudio fue evaluar el efecto del abono orgánico acelerado en el crecimiento y desarrollo de plantas de café (Coffea arábica L.), variedad Catimor, a través de las variables altura de planta, número de hojas, diámetro de tallo, número de ramas por planta, ancho de hoja, concentración de nitrógeno, fósforo y calcio en la hoja.

\section{MATERIALES Y MÉTODOS}

El experimento fue conducido en el Invernadero del Centro Experimental La Molina y Laboratorio Multipropósito del Instituto Nacional de Innovación Agraria (INIA), distrito de La Molina, departamento de Lima, Perú. Localizado a $12^{\circ} 4^{\prime} 36,03^{\prime}$ ' S y $76^{\circ} 56^{\prime}$ $42,857^{\prime \prime}$ O con una altitud de 112 m.s.n.m.

El material vegetal utilizado fue de la especie de café (Coffea arabica L.), variedad Catimor, obtenida de Villa Rica, productor de semillas de café, con registro número 0042014 INIA. El experimento se condujo en invernadero bajo iguales condiciones de manejo, temperatura, humedad e iluminación. Se utilizaron bolsas de polietileno, de $22 \mathrm{~cm}$ de profundidad x $29 \mathrm{~cm}$ de diámetro, con sustrato esterilizado y libre de contaminantes.

El abono orgánico acelerado, denominado "Huallquin Fer", es un biofertilizante producido en la localidad de Hualquin Grande, Tarma, Junín, con el objetivo de disminuir los costos de producción agraria y la contaminación ambiental, por el uso del suero láctico en su elaboración. El abono orgánico acelerado es obtenido a través de una fermentación homolactica de 7 a 10 días, está constituido por estiércol fresco de vacuno (40\%), melaza (10\%), suero láctico (40\%) y el consorcio microbiano Biolac (10\%).

El diseño experimental utilizado fue completamente al azar, con cinco repeticiones, cada tratamiento estaba conformado por cuatro plantas. Los tratamientos fueron: T1 (sin abono orgánico acelerado) y T2 (con abono orgánico acelerado), se realizaron cinco aplicaciones vía foliar, cada 15 días, a una dosis de 20 $\mathrm{ml}$ de abono orgánico acelerado/litro de agua. Para evaluar el efecto del abono orgánico acelerado en el crecimiento y desarrollo de plantas de café (Coffea arabica L.), variedad Catimor. Se evaluaron las siguientes variables: altura de la planta $(\mathrm{cm})$, se midió con una wincha, desde el cuello de la planta hasta el ápice de la hoja bandera del tallo (Figura 1a); número de ramas por planta, fue por conteo; diámetro del tallo, ancho y longitud de hoja $(\mathrm{cm})$, se midió con un calibrador vernier digital (Figura 1b); el porcentaje de materia seca (\%), concentración de nitrógeno, fósforo, potasio y calcio en las hojas (\%), se obtuvo mediante el análisis foliar realizado en el Laboratorio de análisis de suelos, plantas, aguas y fertilizantes de la Universidad Nacional Agraria La Molina. De cada planta se tomaron cuatro hojas del tercio medio (hojas maduras para su evaluación).



Figura 1. Evaluación de características cuantitativas, altura de planta (a) y diámetro de tallo (b) en el tratamiento con abono orgánico acelerado. 
Los resultados obtenidos fueron procesados $\mathrm{y}$ analizados por el programa SAS Versión 8. En los parámetros evaluados se realizó el análisis estadístico multifactorial y la Prueba de Tukey $(\mathrm{P} \leq 0,05)$ para comparar las medias.

\section{RESULTADOS Y DISCUSIÓN}

o se observaron diferencia estadística significativa en los valores altura de planta, diámetro de tallo, ancho de hoja, longitud de hoja, concentración de materia seca, en concentración de nitrógeno, fósforo, potasio y calcio en la hoja (Tabla 1). A pesar de que no hubo efecto significativo en los factores evaluados, los mayores valores obtenidos son del cultivo de café fertilizado con abono orgánico acelerado (Tabla 2).

En tanto fue posible encontrar diferencias significativas $(p<0,05)$ en el número de ramas por planta entre tratamientos (Figura 2). Gardiazábal et al. (2007) indicaron que fertilizar a nivel foliar no se encuentra diferencias estadísticas significativas entre tratamientos. Para la altura al final del experimento, los resultados mostraron que no hay diferencias significativas $(\mathrm{p}>0,05)$ entre los tratamientos.
Estos resultados concuerdan con Mosquera et al. (2016) quienes al evaluar la fertilización orgánica en cafeto (Coffea arabica), al momento del trasplante, obtuvieron plantas de café con altura homogénea entre los tratamientos. Conforme a González et al. (2014), la fertilización contribuye con el óptimo crecimiento y el máximo potencial productivo en el cultivo del café. Zamora et al. (2008) al evaluar el uso de abonos orgánicos obtuvieron mayor altura de planta, número de tubérculos, número de tallos y peso de tubérculos en comparación con los tratamientos que recibieron fertilización química. Boudet et al. (2017) en estudios realizados en tomate determinaron que fertilizar la planta de tomate con fertilizante orgánico, incrementa el rendimiento y sus componentes. Montes (2019) concluye que la fertilización de café con abono orgánico líquido fermentado aerobicamente al $4 \%+$ inoculantes micorrizicos aumentó el rendimiento en el 44,6 en graneo y primera cosecha. Asimismo, utilizar biofertilizantes en el cultivo, hace más eficiente el uso de nutrientes a través de las prácticas de conservación y reducción de pérdidas en campo; mediante el reciclaje de nutrientes orgánicos y el acceso a fuentes de nitrógeno (Gutiérrez-Castorena et al., 2015).

Tabla 1. Análisis de variancia para datos cuantitativos y concentraciones foliar de nutrientes en plantas de café a nivel de vivero.

\begin{tabular}{lccccc}
\hline Tratamientos & \multicolumn{2}{c}{ Variables cuantitativas } \\
\cline { 2 - 6 } & $\begin{array}{c}\text { Altura de planta } \\
(\mathbf{c m})\end{array}$ & $\begin{array}{c}\mathbf{N}^{\circ} \mathbf{d e} \text { ramas } \\
\text { por planta }\end{array}$ & $\begin{array}{c}\text { Diámetro de tallo } \\
\text { principal }(\mathbf{m m})\end{array}$ & $\begin{array}{c}\text { Ancho de hoja } \\
(\mathbf{m m})\end{array}$ & $\begin{array}{c}\text { Longitud de hoja } \\
(\mathbf{m m})\end{array}$ \\
\hline Sin biol & $24,142 \mathrm{a}$ & $1,500 \mathrm{~b}$ & $5,412 \mathrm{a}$ & $5,422 \mathrm{a}$ & $13,354 \mathrm{a}$ \\
Con biol & $24,762 \mathrm{a}$ & $3,250 \mathrm{a}$ & $5,742 \mathrm{a}$ & $5,422 \mathrm{a}$ & $12,952 \mathrm{a}$ \\
CV & 7,797 & 33,287 & 3,590 & 3,693 & 8,358 \\
P-valor & 0,621 & 0,008 & 0,153 & 1,000 & 0,579 \\
\hline Tratamientos & Concentraciones $(\mathrm{CC})$ de nutrientes en las hojas de café $(\%)$ & & CC de \\
& Materia seca & CC & CC de & CC de & Calcio -Ca \\
\hline Sin biol & \multicolumn{7}{c}{ de nutrientes } & Fosforo-P & Potasio- K & 1,558 a \\
Con biol & $26,752 \mathrm{a}$ & $2,156 \mathrm{a}$ & $0,1440 \mathrm{a}$ & $1,844 \mathrm{a}$ & $1,610 \mathrm{a}$ \\
CV & $26,258 \mathrm{a}$ & $2,332 \mathrm{a}$ & $0,1680 \mathrm{a}$ & $1,764 \mathrm{a}$ & 8,087 \\
P-valor & 2,982 & 15,298 & 20,016 & 12,202 & 0,539 \\
\hline
\end{tabular}


Tabla 2. Evaluación de datos cuantitativos y concentraciones café a nivel de vivero (media terror estándar).

\begin{tabular}{|c|c|c|c|c|c|}
\hline \multirow[t]{2}{*}{ Variables } & \multicolumn{3}{|c|}{ T1 (sin abono orgánico acelerado) } & \multicolumn{2}{|c|}{ T2 (con abono orgánico acelerado) } \\
\hline & Promedio & \multicolumn{2}{|c|}{ Error estándar } & Promedio & Error estándar \\
\hline Altura de planta $(\mathrm{cm})$ & 24,142 & \pm & 0,617 & 24,762 & $\pm \quad 1,036$ \\
\hline Número de ramas por planta & 1,500 & \pm & 0,426 & 3,250 & $\pm \quad 0,262$ \\
\hline Diámetro de tallo principal $(\mathrm{cm})$ & 5,412 & \pm & 0,182 & 5,742 & $\pm \quad 0,103$ \\
\hline Ancho de hoja $(\mathrm{cm})$ & 5,422 & \pm & 0,086 & 5,422 & $\pm \quad 0,093$ \\
\hline Longitud de hoja $(\mathrm{cm})$ & 13,354 & \pm & 0,208 & 12,952 & $\pm 0,663$ \\
\hline Variables & \multicolumn{3}{|c|}{ T1 (sin abono orgánico acelerado) } & \multicolumn{2}{|c|}{ T2 (con abono orgánico acelerado) } \\
\hline & Promedio & & Error estándar & Promedio & Error estándar \\
\hline Materia seca & 26,752 & \pm & 0,441 & 26,258 & $\pm \quad 0,236$ \\
\hline Nitrógeno & 2,156 & \pm & 0,194 & 2,332 & $\pm \quad 0,097$ \\
\hline Fósforo & 0,144 & \pm & 0,012 & 0,168 & $\pm \quad 0,016$ \\
\hline Potasio & 1,844 & \pm & 0,129 & 1,764 & $\pm \quad 0,052$ \\
\hline Calcio & 1,558 & \pm & 0,058 & 1,610 & $\pm \quad 0,057$ \\
\hline
\end{tabular}

En relación al número de ramas por planta, se obtuvo mayores ramas en el tratamiento con la aplicación del abono orgánico acelerado con 3,25 ramas por planta, siendo superior estadísticamente al que no se aplicó abono orgánico acelerado con 1,5 ramas. Dias y Melo (2009), reportaron que, luego de probar diversos abonos orgánicos, la mayor cantidad de hojas fue de 6,41 pares. Lao (2013) señala que altas dosis de nitrógeno incrementan el desarrollo vegetativo (mayor contenido de hojas y ramas) aunque disminuyen la producción, mientras que dosis bajas afectan negativamente el desarrollo vegetativo y la producción. Linares-Gabriel et al. (2017) indican que la aplicación foliar de biol tiene un efecto positivo en el área foliar. De acuerdo a Montoya-Restrepo et al. (2017) el área foliar se relaciona con la tasa fotosintética, evapotranspiración y desarrollo vegetativo (Valbuena et al., 2016), así como con la absorción de agua y nutrimentos. El resultado se podría deber a la función que tiene la hoja, de ser el órgano de la planta capaz de aprovechar los nutrimentos que les son aplicados por aspersión (Tisdale et al., 1985).

Según Trinidad \& Aguilar (1999) los nutrientes presentes en el biol, cuando se aplica vía foliar, provocan un efecto en las primeras fases de crecimiento del cultivo, favoreciendo la incorporación de los elementos esenciales en los metabolitos que se generan en la fotosíntesis. He et al. (2000) mencionan que al aumentar el tamaño de la hoja incrementa la eficiencia fotoquímica. Linares-Gabriel et al. (2017) en estudios realizado en Heliconia psittacorum cv. Tropica concluye que el número de hojas fue mayor al testigo y el resto de tratamientos en la fertilización de biol al suelo. Por ser un abono con material orgánico que no está totalmente transformado, su liberación de nutrientes es lenta (Boudet et al., 2015). Orozco (2016) y Castellanos et al. (2015) reportaron que fertilizar con biol u abono orgánico se logra mejorar la calidad de los suelos, por el aporte de nutrientes y microorganismos al suelo, que fijan el carbono, mejoran la capacidad de absorción de agua, promueven las actividades fisiológicas y estimulan el desarrollo de las plantas a través de la producción de enzimas. Similar resultado fue reportado por Boudet et al. (2015) en estudios realizados en plántulas de tomate, donde las variables de crecimiento aumentaron significativamente cuando se utilizó humatos de vermicomposta (Reyes et al., 2015; Torres et al., 2016). Saharan y Nehra (2001) indican que los biofertilizantes influyen en la síntesis de reguladores de crecimiento, como auxinas y giberelinas, que incrementan el desarrollo de pelos radicales y la densidad de la raíz, incrementando la capacidad de absorción del agua y captación de nutrientes, dando a la planta mayor viabilidad, productividad y resistencia a condiciones adversas como sequía. Se debe tener en cuenta la sobrevivencia y compatibilidad de los microorganismos presentes en un biol, existiendo compatibilidad entre Metarrhizium, Trichoderma y Beauveria e incompatibilidad entre los 
generos Paecilomyces y Beauveria (Villacís-Aldaz et al., 2016).

Para la variable, diámetro de tallo, no se observaron efectos significativos entre tratamientos (Tabla 2). Jara (2017) y Julca et al. (2015) obtuvieron mayor diámetro del tallo en el cultivo de café con fertilización en comparación con el testigo, sin mostrar diferencias estadísticas entre los tratamientos. Julca et al. (2002) reportaron, con el uso de abono orgánico, un diámetro de 3,13 mm al final de su ensayo, mientras que Tristão et al. (2006) obtuvieron un valor máximo de 3,8 mm de diámetro de tallo.

Al analizar la concentración de nutrientes en las hojas, el tratamiento con abono orgánico acelerado presentó la mayor concentración de materia seca ,nitrógeno, fósforo, potasio y calcio ( $\mathrm{g} / \mathrm{kg}$ ) en comparación con el tratamiento sin abono orgánico acelerado (Tabla 2). El resultado obtenido se puede deber a la aplicación de nutrientes a través del tejido foliar, que es el órgano donde se concentra la mayor actividad fisiológica de la planta (Rodríguez, 2017; Aguilar, 2016). El uso de productos orgánicos tiene un efecto favorable en el aumento de peso fresco y seco (Valencia, 1972). La planta presenta buen aspecto visual cuando sus tejidos contienen los nutrientes en cantidades y proporciones adecuadas (Malavolta et al., 1997). Sin embargo, a pesar de no existir diferencias estadísticas significativas entre los tratamientos, las hojas del tratamiento 1 presentan deficiencia de nitrógeno (Martinez et al., 2014). Lo cual se puede determinar mediante la concentración de nutrientes en hojas que es una herramienta para el diagnóstico nutricional (PuentesPáramo et al., 2016). En la actualidad son pocos los estudios que se han realizado sobre la relación entre la concentración de nutrientes de la fuente de fertilización y la hoja. Rodríguez (2017) indica que la velocidad de absorción foliar de los diferentes nutrientes es diferente. Gross (1992) sostiene que el nitrógeno se absorbe entre 1 a $6 \mathrm{~h}$, el potasio entre 10 a $24 \mathrm{~h}$, los elementos secundarios y micronutrientes ( $\mathrm{Ca}, \mathrm{Mg}, \mathrm{Fe}, \mathrm{MN}$ y $\mathrm{Zn}$ ) se absorben de 1 a 24 h. Shalajabadi y Salamanca (2016) concluyen que la concentración de los micronutrientes en la formación del fruto presenta el siguiente orden: $\mathrm{Mn}>\mathrm{Fe}>\mathrm{B}>\mathrm{Cu}>\mathrm{Zn}$. Sin embargo, no hubo una relación clara entre las variaciones de los micronutrientes foliares y su demanda por los frutos. A excepción de Mn, no se detectó el efecto de la fertilidad del suelo en la concentración de los elementos en el fruto o en la hoja.

\section{CONCLUSIONES}

De los resultados obtenidos en la presente investigación se concluye que, realizar la fertilización foliar a las plantas de café, con $20 \mathrm{ml}$ de abono orgánico acelerado por litro de agua, incrementa el número de ramas por planta en comparación con el que no es fertilizado con abono orgánico acelerado. Además de incrementar la altura de planta, diámetro de tallo, número de ramas por planta, ancho de hoja, concentración de nitrógeno, fósforo y calcio, pero sin diferencia estadística con el tratamiento sin aplicar abono orgánico acelerado. Se recomienda realizar estudios sobre el efecto del abono orgánico acelerado en la producción de botones florales, inducción de yemas en café o inducción de auxinas, giberalinas y citoquininas (fitohormonas).

\section{Agradecimientos}

Esta investigación fue apoyada por el Programa Nacional de Innovación Agraria (PNIA), en alianza estratégica con la Universidad Nacional Agraria la Molina (UNALM), con el proyecto 119_PTT "Alternativas productivas que generen ingresos económicos elaborando Abono Orgánico Acelerado a partir de excretas de vacuno y suero de leche, y disminución de costos de producción agraria en la localidad de Huallquin Grande - Tarma - Junín”.

\section{Conflictos de intereses}

Los autores firmantes del presente trabajo de investigación declaran no tener ningún potencial conflicto de interés personal o económico con otras personas $\mathrm{u}$ organizaciones que puedan influir indebidamente con el presente manuscrito.

\section{Contribuciones de los autores}

Preparación y ejecución: RCDLC, ARZ, EAR, VPP; Desarrollo de la metodología: RCDLC, ARZ, EAR, VPP; Concepción y diseño: RCDLC, ARZ, EAR, VPP; Edición del artículo: RCDLC, ARZ, EAR, VPP; Supervisión del estudio: RCDLC, ARZ, EAR, VPP. 


\section{LITERATURA CITADA}

- Albaugh, T., Rubilar, R., Fox, R., Allen, L., Urrego, J., Zapata, M., \& Stape, J. (2015). Response of Eucalyptus grandis in Colombia to midrotation fertilization is dependent on site and rate but not frecuency of application. Forest Ecology and Management, 350: 30- 39.

- Aguilar, C., Alvarado, F., Martínez, J., Gutiérrez, A., \& Morales, J. (2016). Evaluación de tres abonos orgánicos en el cultivo de café (Coffea arabica L.) en etapa de vivero. Siembra, 3: 11-20.

- Avelino, J. et al. (2005). Effects of slope exposure, altitude and yield on coffee quality in two altitude terroirs of Costa Rica, Orosi and Santa María de Dota. Journal of the Science of Food and Agriculture, 85 (11): 1869-1876.

- Ballesteros, LF., Teixeira, JA., \& Mussatto, S. (2014). Propiedades químicas, funcionales y estructurales de los granos de café gastados y la piel plateada del café. Journal Food Bioprocess Technol, 7(2): 3493-3503.

- Boudet, A., Chinchilla, V., Boicet, T., \& González, G. (2015). Efectos de diferentes dosis de abono orgánico tipo bocashi en indicadores morfológicos y productivos del cultivo de pimiento (Capsicum annuит L.) var. California Wonder. Revista Centro Agricola, 42(4): 5-9.

- Boudet, A., Tony, F., Santos, R., \& Meriño, Y. (2017). Efecto sobre el tomate (Solanum lycopersicum L.) de diferentes dosis de abono orgánico bocashi en condiciones agroecológicas. Revista Centro Agrícola, 44 (4): 37-42.

- Castellanos, D.E., Rincón, J.M., \& Arguello, A. (2015). Evaluación del efecto de un biofertilizante ligado a un soporte orgánico mineral en un cultivo de lechuga en la Sabana de Bogotá bajo condiciones de invernadero. Revista Colombiana de Ciencias Hortícolas, 9(1): 72-85.

- Castro-Tanzi, S., Dietsch, T., Urena, N., Vindas, L., \& Chandler, M. (2012). Analysis of management and site factors to improve the sustainability of smallholder coffee production in Tarrazu, Costa Rica. Agric. Ecosyst. Environ., 155:172-181.

- Castro-Tanzi, S. (2017). El calcio es un nutriente limitante en cafetales bajo manejo intensivo de fertilizantes en ultisoles. Agronomía Costarricense, 41(1): 105-119.
- Choquehuanca, C. (2018). Plan de negocio para la comercialización de cafés peruanos a través de una plataforma online en lima metropolitana. Tesis de grado de Maestro en Administración, ESAN, Lima, Perú. 180pp.

- Corral, A., Valverde, M., Martínez, R., Chávez, C., \& Benavides, R. (2016). Propiedades físicas, químicas y biológicas de un suelo con biofertilización cultivado con manzano Terra Latinoamericana, 34(4): 441-456.

- DaMatta, F.M., Ronchi, P.C., Maestri, M., \& Barros, S.R. (2007). Ecophysiology of coffee growth and production. Brazilian Journal of Plant Physiology, 19: 485-510.

- Davies, AP., Govaerts, R., Bridson, DM., \& Stoffelen, P. (2006). An annotated taxonomic conspectus of genus Coffea (Rubiaceae). Bot. J. Linn. Soc., 152:465-512.

- Dias, R., \& Melo, B. (2009). Proporção de material orgânico no substrato artificial para a produção de mudas de cafeeiro em tubetes. Revista Ciências Agrotec Lavras, 33(1): 144- 152.

- Gardiazábal, F., Mena, F., \& Magdahl, C. (2007). Efecto de la fertilización en base A N-P-K-Ca-Mg$\mathrm{B}-\mathrm{Zn}$ en palto (Persea americana Mill.) CV. Hass sobre su desarrollo, productividad y postcosecha de la fruta. Proceedings VI World Avocado Congress (Actas VI Congreso Mundial del Aguacate) 2007. Viña Del Mar, Chile. 12 - 16 Nov. 2007. Disponible en: https://www.researchgate.net/publication/2376 91999_EFECTO_DE_LA_FERTILIZACION_EN _BASE_A_N-P-K-Ca-Mg-B-

Zn_EN_PALTO_Persea_americana_Mill_CV_HA SS_SOBRE_SU_DESARROLLO_PRODUCTIVI DAD_Y_POSTCOSECHA_DE_LA_FRUTA/link/ 5589fca508ae9076016fb13c/download

- González, H., Sadeghian; S., \& Jaramillo, A. (2014). Épocas recomendables para la fertilización de cafetales .Avances técnicos Cenicafé Número 422.

- Gross. (1992). Abonos: guía práctica de la fertilización (7 ma edición) Edición. Mundi Prensa. España.

- Gutiérrez-Castorena, EV., Gutiérrez-Castorena; MC., \& Ortiz-Solori, CA. (2015). Manejo integrado de nutrientes en sistemas agrícolas intensivos: revisión. Revista mexicana de ciencias agrícolas, 6(1): 201-15. 
- He, J., Pheng, T.L., \& Chong, J.G. (2000). Alleviation of photoinhibition in heliconia grown under tropical natural conditions after release from nutrient stress. Journal of Plant Nutrition, 23(2): 181-196.

- Jara, D. (2017). Efecto de dos fuentes de materia orgánica en la producción de plantones de café (Coffea arabica) en el caserío nuevo Amazonas, distrito Yamón, provincia Utcubamba - Amazonas. Tesis de Ingeniero Agrónomo, Universidad Nacional "Toribio Rodríguez de Mendoza de Amazonas, Chachapoyas, Perú. 61 pp.

- Julca, A., Borjas, R., Bello, S., Ladera, Y., \& Rebaza, D. (2015). El crecimiento del café var. caturra roja y su relación con la aplicación de abonos orgánicos. Revista de la Facultad de Ingeniería de la USIL, 2 (2):75-89.

- Julca, A., Solano, W., \& Crespo, R. (2002). Crecimiento de Coffea arabica variedad Caturra amarillo en almácigos con substratos orgánicos en Chanchamayo, selva central del Perú. Investigación Agraria: Producción Protección Vegetal, 17(3): 353-365.

- Kadri, SM., Zaluski, R., Lima, GPP., Mazzafera, P., \& Orsi, RD. (2016). Characterization of Coffea arabica monofloral honey from Espirito Santo, Brazil. Food Chemistry, 203:252-257.

- Kathurima, C.W., Gichimu, B.M., Kenji, G.M., Muhoho, S.M., \& Boulanger, R. (2009). Evaluation of beverage quality and green bean physical characteristics of selected Arabica coffee genotypes in Kenya. African Journal of Food Science, 3:365371.

- Lammel, D.R., Azevedo, L.C.B., Paula, A.M., Armas, R.D., Baretta, D., \& Cardoso, E. J.B.N. (2015). Microbiological and faunal soil attributes of coffee cultivation under different management systems in Brazil. Brazilian Journal of Biology, 75(4): 894-905.

- LAO, O. (2013). Fertilización en el cultivo de palto. Guía técnica. Universidad Nacional Agraria la Molina - AGROBANCO.

- Linares-Gabriel, A., López-Collado, C.J., TinocoAlfaro, C.A., Velasco-Velasco, J., \& LópezRomero, G. (2017). Application of biol, inorganic fertilizer and superabsorbent polymers in the growth of heliconia (Heliconia psittacorum cv. Tropica).
Revista Chapingo Serie Horticultura, XXIII (1): 3548.

- López, W., Castro, I., Salinas, E., Reynoso, E., \& López, J. (2016). Propiedades de los suelos cafetaleros en la Reserva de la Biósfera El Triunfo, Chiapas, México. Revista Mexicana de Ciencias Agrícolas, 7(3): 607-618.

- Malavolta, E., Vitti, G.C., \& De Oliveira, S.A. (1997). Avaliação do estado nutricional das plantas. Principios e aplicações. 2a edicicão. Piracicaba. Associação Brasileira para Pesquisa da Potassa e do Fosfato, Brasil.

- Martínez, C., Muena, V., \& Ruiz, R. (2014). Nutrición y Fertilidad en Palto. Boletín INIA $N^{\circ}$ 283, 74 p. Instituto de Investigaciones Agropecuarias, La Cruz, Chile. Disponible en: http://biblioteca.inia.cl/medios/biblioteca/boletines/ NR40071.pdf

- Montes, C. (2019). Efecto de la fertilización con abono orgánico (A.L.O.F.A) en plantas de cafè (coffea arábica). Localización: Scientia et Technica, 24 (2): 331-339.

- Montoya-Restrepo, E., Hernández-Arrefondo, J., Unigarro-Muñoz, C., \& Flórez-Ramos, C. (2017). Estimación del área foliar en café variedad Castillo a libre exposición y su relación con la producción. Cenicafé, 68: 55-61.

- Mosquera, A., Melo, M., Quiroga, C., Avendaño, D.M., Barahona, M., Galindo, F.D., Lancheros, J.J., Prieto, S.A., Rodriguez, A., \& Sosa, D. (2016). Evaluación de fertilización orgánica en cafeto (Coffea arabica) con pequeños productores de Santander, Colombia. Temas Agrarios, 21(1): 90 101.

- Orozco, A.L., Valverde, M.I., Martínez, R. Chávez, C., \& Benavides, R. (2016). Propiedades físicas, químicas y biológicas de un suelo con biofertilización cultivado con manzano. Terra Latinoamericana, 34 (4): 441-456.

- Puentes-Páramo, Y., Menjivar-Flores, JC., \& Aranzazu-Hernández, F. (2016). Concentración de nutrientes en hojas, una herramienta para el diagnóstico nutricional en cacao. Agron. Mesoam. , 27(2): 329-336.

- Ramírez, F., Boniche, J., Bertsch, F., \& Mora, L. (2002). Consumo de nutrimentos por los frutos y bandolas de café caturra durante un ciclo de 
desarrollo y maduración en Aquiares, Turrialba, Costa Rica. Agronomía Costarricense, 26:33-42.

- Reyes, J., Torres, J., Murillo, B., Herrera, M., Guridi, F., Luna, R., López, R., \& Real, G. (2015). Humatos de vermicompost y su efecto en el crecimiento de plántulas de tomate (Solanum lycopersicum, L). Biotecnia, XVII (2): 9 -12.

- Rodríguez, A. (2017). Estudio de la aplicación foliar de potasio en la prevención de potasio en la prevención de la caída de frutos Persea americana Mill. Var. Hass en Casma, Ancash. Tesis de Ingeniero agrónomo, Universidad de Trujillo, Perú. 43 pp.

- Sadeghian, K.S., \& González, O.H. (2012). Alternativas generales de fertilización para cafetales en etapa de producción. Avances técnicos Cenicafé, 423: 1-4.

- Saharan, B.S., \& Nehra, V. (2001). Plant growth promoting rhizobacteria: A critical review. Life Sci. Med. Res. 2011: LSMR-21.

- Salazar, G., \& Sadeghian, K. (2016). Respuesta del café (Coffea arabica L.) a la fertilización antes y después de la Zoca. Cenicafé, 67(1) 81-93.

- $\quad$ Tisdale, S.W., Nelson, W.L., \& Beaton, J.D. (1985). Soil fertility and fertilizers. New York, USA: MacMillan Publishing Co.

- Trinidad, S.A., \& Aguilar, M.D. (1999). Fertilización foliar, un respaldo importante en el rendimiento de los cultivos. Terra, 17(3): 247-255.

- Tristão, F., De Andrade, S. \& Silveira, A. 2006. Fungos micorrízicos arbusculares na formação de mudas de cafeeiro, em substratos orgânicos comercias. Bragantia, 35(4): 649-658.

- Torres, J., Reyes, J., \& González, J. (2016). Efecto de un bioestimulante natural sobre algunos parámetros de calidad en plántulas de tomate (Solanum lycopersicum, L). Biotecnia, XVIII (2): 11-15.

- Valencia, G. (1972). Utilización de la pulpa de café en los Almácigos En Avances Técnicos de Cenicafé Tomo 1. Federación de Cafeteros de Colombia. Chinchina. Colombia. 21-22pp.

- Valbuena, N., Parraga, P., Linares, L., Ramos, J., \& Junco, J. (2016). Modelos de estimación de área foliar a partir de observaciones morfológicas en Brachiaria brizantha cv. Toledo. Revista Unellez de Ciencia y Tecnología, 34: 40-44.
- Villacís-Aldaz, L., Chungata, L., Pomboza, P., \& León, O. (2016). Compatibilidad y tiempo de sobrevivencia de cuatro microorganismos benéficos de uso agrícola en biol. Journal of the Selva Andina Biosphere, 4(1): 39-45.

- Wang, N., Jassogne, L., van Asten, PJA., Mukasa, D., Wanyama, I., Kagezi, G., \& Giller, KE. (2015). Evaluating coffee yield gaps and important biotic, abiotic, and management factors limiting coffee production in Uganda. European Journal of Agronomy, 63:1-11.

- Zamora, F., Tua, D., \& Torres, D. (2008) Evaluación de cinco fuentes orgánicas sobre el desarrollo vegetativo y rendimiento del cultivo de papa. Agronomía Trop., 58(3): 233-243.. 